\title{
KNOWLEDGE AND RESPONSIBILITY OF THE PARENTS TOWARD CHILD'S ORAL HEALTH: A CROSS-SECTIONAL STUDY
}

\author{
SHASMITHA R, SHANMUGAAVEL AK*
}

Department of Pediatric and Preventive Dentistry, Saveetha Dental College and Hospitals, Chennai - 600 095, Tamil Nadu, India. Email: dentistshan@gmail.com

Received: 09 August 2016, Revised and Accepted: 15 October 2016

\section{ABSTRACT}

Objective: The aim of this study was to understand the dental knowledge and responsibility of the parents toward child's oral health in a dental office.

Methods: A questionnaire (Annexure A) was distributed to 200 parents who came to Saveetha Dental College and Hospitals. Parent's information regarding dental knowledge, responsibility taking, and knowledge regarding child's oral health was collected with the aid of the structured questionnaire.

Result: The results showed that the dental knowledge of parents is lower than that of the experts. Parents mentioned that fluoride has less importance in enamel protection $(\mathrm{p}=0.87)$ than the experts $(\mathrm{p}<0.001)$. Almost $100 \%$ of the parents were engaged in the daily assistance of tooth brushing for their children. However, child's diet control is poor. Nearly $26 \%$ of the parents have no control of sweet consumption for their child. About $45 \%$ of the parents have no control of biscuits consumption for their child. Moreover, $22 \%$ of the parents of uncooperative child dental patients mentioned that it is a dentist's responsibility for the unwillingness of the treatment and treatment refusal stated by the child.

Conclusion: This study showed that the parents have poor dental knowledge and are not responsible for their child's oral health. They do not maintain proper dietary habits for their children which may lead to the increased incidence of caries in childhood. Hence, there is a need for increase in dental knowledge of the parents to maintain a proper oral health of their children.

Keywords: Parental behavior, Parental dental knowledge, Child's behavior.

(C) 2017 The Authors. Published by Innovare Academic Sciences Pvt Ltd. This is an open access article under the CC BY license (http://creativecommons. org/licenses/by/4. 0/) DOI: http://dx.doi.org/10.22159/ajpcr.2017.v10i1.14568

\section{INTRODUCTION}

Pediatric dental practitioners were expected to recognize and effectively treat childhood dental diseases that are within the knowledge and skills acquired during their professional education. Parent's dental knowledge has an indirect influence on child's behavior in a dental office. Parental guidance is the process by which practitioners help patients identify appropriate and inappropriate behavior, learn problem-solving strategies, and develop impulse control, empathy, and self-esteem. This process is a continuum of interaction involving the dentist and dental team, the patient, and the parent; its goals are to establish communication, alleviate fear, and anxiety, deliver quality dental care, build a trusting relationship between dentist and child and the parent, and promote the child's positive attitude toward oral health care [1]. Safe and effective treatment of these diseases requires understanding of parent's knowledge and responsibility and, at times, modifying the child's and family's response to care. Hence, parent's dental knowledge and responsibility toward child's oral health is studied. One of the most widely used systems was introduced by Frankl in 1962. It is referred to as the Frankl behavioral rating scale. The scale divides observed behavior into four categories, ranging from definitely positive to definitely negative. It is often considered the gold standard in clinical rating scales, mainly as a result of its wide usage and acceptance in pediatric dentistry research.

\section{METHODS}

A questionnaire (Annexure A) was distributed to 200 pedodontic patient's parents, who came to Saveetha Dental College and Hospitals being randomly selected. Parent's information regarding dental knowledge, responsibility taking, and knowledge regarding child's oral health were collected with the aid of the structured questionnaire along with the parent's consent attached to it. The questionnaire was presented in a way that both urban and rural populations can acknowledge the study with ease. A child's oral health was examined clinically by the dental practitioners in Saveetha Dental College and recorded in the form of Indices - DMFT, PUFA. The behavior of the child during the dental treatment has been recorded according to the Frankl behavioral rating scale [2].

\section{Inclusion criteria}

All pedodontic patients between the age of 4 and 15 years were included in the study.

\section{Exclusion criteria}

All pedodontic patients with communication difficulties and congential disoreders were excluded from the study.

\section{Instruments}

Parental dental knowledge: Ratings of the importance of enamel protecting agent and five caries etiological factors were made on a 4 -point scale from 1 to 4 (Tables 1 and 2).

Children's oral health behavior as perceived by the parents: Children's tooth brushing and eating habits were reported with a series of 4-step questions made on a 4-point scale from 1 to 4 .

Parental responsibility: Seven common situations in children's dentistry were described and the parents answered on a 4-point scale from " 1 - totally a responsibility of a dentist" to " 4 - entirely the family's responsibility" (Fig. 1). 
Behavioral score of the child during the dental treatment is recorded using the Frankl behavioral Ranking scale. The scale classifies child behavior as follows: Category \#1 - Definitely negative, Category \#2 - Negative, Category \#3 - Positive, and Category \#4 - Definitely positive.

\section{Statistical analysis}

Differences between groups were analyzed using descriptive statistics and non-parametric methods for two independent groups. Chi-square tests were used to analyze differences in proportions. $\mathrm{p}$ values were calculated using one-way ANOVA and Mann-Whitney U-test.

\section{RESULTS}

A total of 200 parents of children aged between 4 and 15 years were participated in this study. Children with lower age group have the lowest behavioral scores compared to the children of older age group. Among the $11 \%$ of children with the least behavioral score, $10 \%$ of children belonged to the lower age group between 4 and 6 years of age.

\section{Parental dental knowledge}

The results showed that the dental knowledge of parents is lower than that of the experts. Parents mentioned that fluoride has less importance in enamel protection $(\mathrm{p}=0.87)$ than the experts $(\mathrm{p}<0.001)$. The correct answers regarding fluoride were given by the parents of children with the least behavioral score (mean value=2.6); for calcium, parents of children with the behavioral score 3 have given the correct answers (mean value=2.25); and for vitamins, parents of children with the behavioral score 2 has given the correct answers (mean value=2.25). All the parents mentioned that inadequate tooth brushing is a major risk factor:

\section{Child's oral health as perceived by the parents}

Almost $100 \%$ of the parents were engaged in the daily assistance of tooth brushing for their children. However, child's diet control is poor. About $26 \%$ of the parents had no control of sweet consumption for their child. Nearly $45 \%$ of the parents had no control of biscuits consumption for their child. Moreover, $73 \%$ of the parents have no control of sugary drinks for their children.

\section{Parent's responsibility}

Nearly $22 \%$ of the parents of uncooperative child dental patients mentioned that it is a dentist's responsibility for the unwillingness of the treatment and treatment refusal stated by the child. About $100 \%$ of the parents mentioned that it their responsibility for the care of tooth brushing and toothache from the restored tooth. Almost $42 \%$ of the parents said that recall intervals can only be given by their dentists whereas $60 \%$ experts said that it is entirely family's responsibility. Almost $16 \%$ of the parents stated that it is the duty of the dentists to identify the early signs of tooth decay whereas $50 \%$ experts mentioned that it is entirely family's responsibility. Nearly $32 \%$ of the parents said that it is entirely dentist's responsibility for the unwillingness of the child dental patient to visit the dentist whereas $90 \%$ experts mentioned that it is entirely family's responsibility. About $27 \%$ of the parents mentioned that it is entirely dentist's responsibility for the cause of child's treatment refusal whereas $90 \%$ experts said that it is entirely family's responsibility. The detailed statistical analyses are shown in Tables $1-3$ and Fig. 1.

\section{DISSCUSSION}

\section{Parent dental knowledge}

According to the descriptive statistic, many parents gave incorrect answers that fluoride has low importance in enamel protection. Parents considered the role of calcium and vitamins have equal importance in enamel protection. This is due to the unawareness that fluoride has high importance in enamel protection. A similar result is also obtained from a study performed in Sweden [3]. This study has reported that poor attitude and dental knowledge of parents toward oral health of infants and young children are associated with increased caries prevalence.

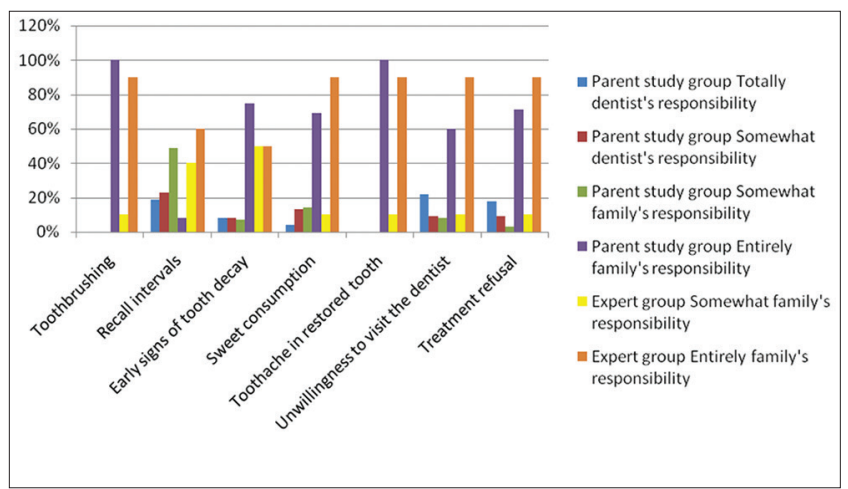

Fig. 1: Parent's responsibility (comparison among parent study group and expert group)

Table 1: Parental dental knowledge (means and SD) compared to the experts

\begin{tabular}{llllllll}
\hline $\begin{array}{l}\text { Enamel } \\
\text { Protecting } \\
\text { agent }\end{array}$ & \multicolumn{2}{l}{ Behavioral score } & & p value & Total (n=200) & Experts (n=10) & $\begin{array}{l}\text { Total versus } \\
\text { experts }\end{array}$ \\
\cline { 2 - 7 } & $\begin{array}{l}\text { Definitely } \\
\text { negative }\end{array}$ & Negative & Positive & $\begin{array}{l}\text { Definitely } \\
\text { positive }\end{array}$ & & & \\
\hline Fluoride & $2.6 \pm 0.94$ & $2.44 \pm 1.15$ & $2.36 \pm 1.11$ & $2.45 \pm 1.19$ & 0.873 & $2.44 \pm 1.1$ & $3.7 \pm 0.3$ \\
Calcium & $2.15 \pm 1.04$ & $2.19 \pm 0.97$ & $2.25 \pm 1.02$ & $2.07 \pm 0.99$ & 0.827 & $2.17 \pm 0.9$ & $2.5 \pm 1.2$ \\
Vitamins & $1.95 \pm 0.68$ & $2.25 \pm 0.85$ & $2.10 \pm 0.83$ & $2.07 \pm 0.99$ & 0.503 & $2.13 \pm 1.0$ & $2.1 \pm 1.0$ \\
\hline
\end{tabular}

*** $\mathrm{p}<0.001 ;{ }^{*} \mathrm{p}<0.05$, P value calculated using one-way ANOVA and Mann-Whitney U-test. SD: Standard deviation

Table 2: Parental dental knowledge regarding caries risk factor (means and SD) compared to the experts

\begin{tabular}{|c|c|c|c|c|c|c|c|c|}
\hline \multirow[t]{2}{*}{ Etiological factors } & \multicolumn{4}{|c|}{ Behavioral score } & \multirow[t]{2}{*}{ p value } & \multirow[t]{2}{*}{ Total $(n=200)$} & \multirow[t]{2}{*}{ Experts $(n=10)$} & \multirow{2}{*}{$\begin{array}{l}\text { Total } \\
\text { versus } \\
\text { experts }\end{array}$} \\
\hline & $\begin{array}{l}\text { Definitely } \\
\text { negative }\end{array}$ & negative & Positive & $\begin{array}{l}\text { Definitely } \\
\text { positive }\end{array}$ & & & & \\
\hline Hereditary & $1.20 \pm 0.41$ & $1.39 \pm 0.60$ & $1.31 \pm 0.62$ & $1.21 \pm 0.50$ & 0.403 & $1.31 \pm 0.56$ & $2.8 \pm 0.6$ & $* * *$ \\
\hline Between meal eating & $2.1 \pm 1.20$ & $2.38 \pm 0.88$ & $2.23 \pm 1.00$ & $2.44 \pm 0.97$ & 0.459 & $2.32 \pm 0.96$ & $3.5 \pm 0.3$ & $* * *$ \\
\hline $\begin{array}{l}\text { Inadequate tooth } \\
\text { brushing }\end{array}$ & 4.00 & 4.00 & 4.00 & 4.00 & 0.00 & 4.00 & $3.8 \pm 0.1$ & \\
\hline Saliva composition & $1.8 \pm 0.89$ & $1.77 \pm 0.831$ & $1.82 \pm 0.847$ & $1.82 \pm 0.796$ & 0.982 & $1.80 \pm 0.82$ & $3.2 \pm 0.6$ & $* * *$ \\
\hline Infection and diseases & $3.2 \pm 0.834$ & $3.28 \pm 0.88$ & $3.21 \pm 0.85$ & $3.56 \pm 0.60$ & 0.084 & $3.33 \pm 0.8$ & $2.8 \pm 0.8$ & $*$ \\
\hline
\end{tabular}

${ }^{* * *} \mathrm{p}<0.001 ;{ }^{*} \mathrm{p}<0.05$, P value calculated using one-way ANOVA and Mann-Whitney U-test. SD: Standard deviation 
Table 3: Children's oral health behavior as perceived by their parents

\begin{tabular}{lll}
\hline \multirow{2}{*}{ Oral hygiene habits } & \multicolumn{2}{l}{ Parent study group (\%) } \\
\cline { 2 - 3 } & No & Yes \\
\hline Daily assistance in tooth brushing & 0.00 & 100.00 \\
Sweets more than one per day & 26.00 & 74.00 \\
Biscuits more than one per day & 45.00 & 55.00 \\
Frequent consumption of sugary drinks & 73.00 & 27.00 \\
\hline
\end{tabular}

All the parents indicated that inadequate tooth brushing is a major caries risk factor. A few parents gave incorrect answers that hereditary has a role in accumulation of caries. There is no sufficient mass media awareness regarding dental knowledge. Young children's oral health maintenance and outcomes are influenced by their parent's knowledge and beliefs, which affect oral hygiene and healthy eating habits. Without basic knowledge of caries risk factors, importance of the deciduous teeth, and oral maintenance, it is difficult to employ effective diseasepreventive strategies [4]. Parent's knowledge and positive attitude toward good dental care are very important in the preventive cycle. Every dentist should interact with the patients and the parents to create a better relationship and provide minimal dental knowledge to them.

Children's oral health behavior as perceived by the parents

All the parents stated that they are engaged in daily assistance of tooth brushing for their children. A few parents stated that sweets and biscuit consumption has no significant effects on dental caries development and majority of the parents stated that sugary drinks have no significance on dental caries development. This shows that they have poor control of their child's diet. However, majority of the parents had good knowledge regarding the role of diet in oral health; they believed that sweets and snacks contribute to caries.

\section{Parent's responsibility}

All the parents were totally responsible for child's oral habits such as regular tooth brushing and pain from the restored tooth. However, the uncooperative act of the child may lead to dislodged or failure of restorations. Moreover, it is a parent's duty to follow the child's activity not to bother the restoration. A few parents stated that it is the duty of the dentists to identify the early signs of tooth decay and inform them to the parents as they are not well aware, but the majority of the experts stated that its initially the responsibility of the parents in identifying the early signs of dental caries. Parents play an active role in every aspect of the child's activity; this also includes them to be an active participant in forming high potential in decision-making and treatment planning with the dentists.

\section{CONCLUSION}

This study shows that the parents have poor dental knowledge and are not responsible for their child's oral health. They do not maintain proper dietary habits for their children which may lead to the increased incidence of caries in childhood. Hence, there is a need for increase in dental knowledge of the parents to maintain a proper oral health of their children.

\section{REFERENCES}

1. American Academy of Pediatric Dentistry. Guideline on behaviour guidance for the pediatric dental patient. Pediatr Dent 2015;37(6):15-6.

2. Goleman J. Cultural factors affecting behavior guidance and family compliance. Pediatr Dent 2014;36(2):121-7.

3. Arnrup K, Berggren U, Broberg AG, Lundin SA, Hakeberg M. Attitudes to dental care among parents of uncooperative vs. Cooperative child dental patients. Eur J Oral Sci 2002;110(2):75-82.

4. Stecksen-Blicks C, Holm AK. Between-meal eating, toothbrushing frequency and dental caries in 4-year-old children in the north of Sweden. Int J Paediatr Dent 1995;5(2):67-72. 


\section{ANNEXURE A}

பெற்றோரின்பெயர்:

பணி:

குழந்தையின் பெயர்:

குழந்தையின் வயது:

1)கீழ்க்கண்டவற்றுள் எது எனாமலைப் பாதுகாக்கும் பொருள் என்று எண்ணுவதைத் தேர்ந்தெடுக:

ளோரைட் — மிகவும் முக்கியம் ( ) முக்கியம் ( ) சிறிதளவு முக்கியம( ) தேவையில்லை( )

கால்சியம் _ ம மிகவும் முக்கியம் ( ) முக்கியம் ( ) சிறிதளவு ம்க்கியம( ) தேவையில்லை( )

வைட்டமின்கள் _ _ மிகவும் முக்கியம் ( ) முக்கியம் ( ) சிறிதளவு முக்கியம( ) தேவையில்லை( )

2) கீழ்க்கண்டவற்றுள் எது முக்கியமான பல்சொத்தை அதிகரிக்கும் காரணிகள் என்று எண்ணுவதைத் தேர்ந்தெடுக:

பரம்பரை _ ம மிகவும் முக்கியம் ( ) முக்கியம் ( ) சிறிதளவு மகுக்கியம் ( ) தேவையில்லை( )

குறைவான பல்துலக்கும் பழக்கம் ( ) முக்கியம் ( ) சிறிதளவு முக்கியம் ( ) தேவையில்லை( )

உமிழ் நீரின் கலவை _ மிகவும் முக்கியம் ( ) முக்கியம் ( ) சிறிதளவு முக்கியம் ( ) தேவையில்லை( )

தொற்று நோய் மற்றும் வியாதி _ மிகவும் முக்கியம் ( ) முக்கியம் ( ) சிறிதளவு முக்கியம் ( ) தேவையில்லை( )

உணவை சாப்பிட்ட இடையே சாப்பிடுவது _ மிகவும் முக்கியம் ( ) முக்கியம் ( ) சிறிதளவு முக்கியம் ( ) தேவையில்லை( )

3) கீழ்க்கண்டவற்றுள் எது குழந்தைகளின் வாய் வழிச்சுகாதாரம் மேம்படுத முக்கியமான காரணி என்று எண்ணுக்க்கிறீர்கள்: 
பல் துலக்க தினமும் உதவிச் செய்தல் — மிகவும் முக்கியம் ( ) முக்கியம் ( ) சிறிதளவு முக்கியம் ( ) தேவையில்லை( )

ஒரு நாளைக்கு ஒன்றுக்கும் மேற்பட்ட இனிப்பலக்காரங்கள் எடுத்துக் கொள்வது _ மிகவும்முக்கியம் ( ) முக்கியம் ( ) சிறிதளவு முக்கியம் ( ) தேவையில்லை( ) ஒரு நாளைக்கு ஒன்றுக்கும் மேற்பட்ட பிஸ்கட் எடுத்துக்கொள்வது மிகவும் ம்க்கியம் ( ) முக்கியம் ( ) சிறிதளவு ம்க்கியம் ( ) தேவையில்லை( ) அடிக்கடி இனிப்புப்பானங்கள் அருந்துவது _ மிகவும் ம்க்கியம் ( ) முக்கியம் ( ) சிறிதளவு முக்கியம் ( ) தேவையில்லை( )

4) கீழ்க்கண்டவற்றுள் எவை முக்கிய பெற்றோரின் கடமை:

குழந்தைக்கு பல் துலக்குவது மிகவும் முக்கியம் ( ) முக்கியம் ( ) சிறிதளவு முக்கியம் ( ) தேவையில்லை( )

மறுநியமனம் மிகவும் முக்கியம் ( ) முக்கியம் ( ) சிறிதளவு முக்கியம் ( ) தேவையில்லை( )

ஆரம்ப சொத்தைப்பல் அடையாளங்கள் மிகவும் முக்கியம் ( ) முக்கியம் ( ) சிறிதளவு முக்கியம் ( ) தேவையில்லை( )

இனிப்புப் பலகாரங்கள் எடுத்துக்கொள்வது மிகவும் முக்கியம் ( ) முக்கியம் ( ) சிறிதளவு முக்கியம் ( ) தேவையில்லை( ) மீட்டெடுத்தப்பட்ட பற்களிருந்து வரும் வலியை கவணிப்பது மிகவும் முக்கியம் ( ) முக்கியம் ( ) சிறிதளவு முக்கியம் ( ) தேவையில்லை( )

பல் மருத்துவரிடம் செல்ல விருப்பமின்மை மிகவும் முக்கியம் ( ) ம்க்கியம் ( ) சிறிதளவு முக்கியம் ( ) தேவையில்லை( )

சிகிச்சை மறுத்தல மிகவும் முக்கியம் ( ) முக்கியம் ( ) சிறிதளவு முக்கியம் ( ) தேவையில்லை( )

பெற்றோரின் கையெழுந்து: 